\title{
Incremental clustering of sonar images using self-organizing maps combined with fuzzy adaptive resonance theory
}

\author{
Ahmed Nait Chabane $^{\mathrm{a}, \mathrm{b}}$, Naveed Islam ${ }^{\mathrm{a}, \mathrm{c}, *}$, Benoit Zerr ${ }^{\mathrm{a}}$ \\ a ENSTA Bretagne, Lab-STICC (UMR CNRS 6285), 2 rue Francois Verny, 29806 Brest Cedex 9, France \\ b LINEACT/CESI, Saint Nazaire, France \\ c Islamia College University Peshawar, Pakistan
}

In this paper we introduce a new unsupervised segmentation algorithm for textured sonar images. A Dynamic Self-Organizing Maps (DSOM) algorithm capable of incremental learning has been developed to automatically cluster the input data into relevant classes of seabed. DSOM algorithm is an extension of classical SelfOrganizing Maps (SOM) algorithm combined with Adaptive Resonance Theory (ART) technique. The proposed approach is based on growing map size during learning processes. Starting with a minimal number of neurons, the map size increases dynamically and the growth is controlled by the vigilance threshold of the ART network. To assess the consistency of the proposed approach, the DSOM algorithm is first tested on simulated data sets and then applied on real sidescan sonar images. The results obtained using the proposed approach demonstrate its capability to successfully cluster sonar images into their relevant seabed classes, very close to those resulting from human expert interpretation.

\section{Introduction}

Image segmentation is an important step in the image analysis chain. It addresses the problem of dividing an images into homogeneous groups of pixels based on a similarity measure. In terms of a priori knowledge, two families of image segmentation algorithms can be distinguished: the supervised and the unsupervised approaches. The supervised algorithms rely on training phase, which is based on a precise and comprehensive a priori knowledge of the type or label of the training data. The widely used supervised algorithms are based on Maximum a Posteriori (MAP) or Maximum Likelihood (ML) technique (Duda et al., 2001).

Seafloor classification is the segregation of sonar images of seabed into separate physical entities or classes. It is very useful and active area of research in the field of seabed mapping, marine geophysics, geological survey, exploring underwater natural resources, marine habitat and underwater acoustics. Similar to the segmentation of ordinary natural images, the segmentation of sonar images with supervised algorithms requires ground truth data. In practice such ground truth is difficult to acquire (underwater video, dredge or core data sampling) and therefore labeling the seabed types often reduces to a few discrete locations. The supervised approach gives satisfactory results only when a comprehensive training set is available. If the training set lacks a particular kind of seabed, it will be unknown to the classifier and the classification will be reduced to the closest known sediment class. As it is not always feasible to have seabed ground truth classes and to know the entire seabed types before the training phase, an unsupervised algorithm capable to determine clusters according to statistical similarity and independently to the expert interpretation is suitable for sonar images. Recent progress in underwater robotics has been aimed at developing autonomous underwater vehicles (AUVs), which allow automatic data collection and interpretation with on board processing techniques and unsupervised algorithms for classification (Wynn et al., 2014). Hence, the unsupervised algorithms can be implemented in real time on these AUVs to fully automate the seabed classification of unknown areas.

The unsupervised approaches exploit the resemblance between statistics features estimated from images, with no a-priori knowledge about data labeling or number of classes. In this case, clustering algorithms are used to gather pixels or regions in similar groups. Approaches to unsupervised learning include: clustering algorithms (e.g., ISODATA, K-means, mixture models and hierarchical clustering) (Hastie et al., 2009; Acharyya, 2008), blind signal separation generally used for dimensionality reduction and features extraction (e.g., Principle Component Analysis (PCA), Independent Component Analysis (ICA)) (Acharyya, 2008) and neural network models using

\footnotetext{
* Corresponding author at: Islamia College University Peshawar, Pakistan.

E-mail addresses: nait.chabane.ahmed@gmail.com (A.N. Chabane), dr.naveedislam2014@gmail.com (N. Islam), benoit.zerr@ensta-bretagne.fr (B. Zerr).
} 
unsupervised learning. Among these models, Self-Organizing Maps (SOM) developed by (Kohonen, 1982) and Adaptive Resonance Theory (ART) developed by (Carpenter and Grossberg, 1988) have been chosen as they have successfully solved many different kinds of problems in various research fields (for example (Kohonen et al., 1996; Carpenter et al., 1998; Kim et al., 2001)).

In this work, a new approach for unsupervised segmentation of sidescan sonar images is proposed. Our approach is based on the mixture of two neural network algorithms: the SOM and ART algorithms. The SOM algorithm is a powerful tool for clustering and data mining. It has been used for mapping high-dimensional data into generally one, two or three dimensional feature map (Kohonen, 2013). One of the important characteristic of SOM algorithm is its ability to preserve the topology of input space using neighborhood function. It means that input data which is similar in term of features distance will be close after projection by SOM algorithm. This topological preservation of data allows best visualization and identification of data clusters. The SOM algorithm is classically presented as two-dimensional (2D) grid of neural nodes. A group of close nodes on the grid is a cluster and represent a certain class of the given data. However, classical SOM algorithm has some limitations i. e. the size of the grid and the number of nodes have to be predetermined, whereas the proposed method dynamically increase the size of the neural map that incrementally characterize the detection of new classes systematically. The problem of determining the size of the grid in SOM depends on the size of the data and the structures of the clusters. In this regard, many approaches exist to determine the size of the grid, for example: Sammon's projections or empirical methods (Sammon, 1969), which are based on the cardinality of the input data (e.g. $5 \sqrt{N}$, where $N$ is the number of observations), another approach given in (Vesanto and Alhoniemi, 2000) is used to create a large grid with additional stages of clustering. But in practice, many experiments and simulation need to be conducted to define the appropriate size of the map. In the case of unknown structure of the data, an incremental or dynamic structure of the grid is suitable.

The remainder of this paper is organized as follows. Section 2 introduces the related works for dynamic neural network. Section 3 reviews the SOM and Fuzzy ART algorithms and then describes the proposed DSOM algorithm for incremental clusters detection. Experimental results are shown in Section 4 and finally conclusion is given in Section 5 .

\section{Related works}

Artificial Neural Networks (ANNs) are computational models (inspired by the functioning of cerebral cortex) which are capable of extracting meaning, detecting trends and patterns in complex data of heterogeneous nature (Hansen and Salamon, 1990). The SOM is one of the well known algorithm of ANN models and it is widely used in numerous applications for visualizing (visualization of high dimensional data into low dimensional views), clustering problems without the knowledge of class memberships and image classification. Several works used SOM algorithm on various fields of research. For example, (Kinnunen et al., 2012) uses the SOM algorithm for unsupervised objects discovery. In remote sensing, for hyperspectral imagery, (Liu et al., 2010) proposed an approach based on SOM and fuzzy membership for decomposition of mixed pixels. Several authors have successfully applied different approaches of ANN to the problem of seafloor classification (Muller et al., 1997; Stewart et al., 1994; Bourgeois and Walker, 1919; Maillard et al., 1992; Vink et al., 2000). Similarly, the use of fuzzy ART algorithm for the segmentation of acoustic image is implemented by (Vink et al., 2000). To overcome the limitation of the fixed size grid of the classical SOM algorithm, several dynamic neural network models have been proposed.

The Neural Gas Algorithm (NGA) developed by (Martinetz et al., 1993) is an unsupervised neural network, which successively add units (or nodes) to an initial small network by evaluating local statistical measures gathered during previous adaptation steps.
Another algorithm called Growing Cell Structures (GCS) developed by (Fritzke, 1994) is based on the basic approach of NGA with fixed topology dimensionality (2-D or 3-D). In (Alahakoon et al., 2000), the authors proposed a Dynamic Self-organizing Maps with controlled growth (GSOM) for knowledge discovery. The advantage of GSOM is the control of the size of the grid using spread factor. The spread factor in this case is independent of data dimensionality and can be used as threshold to create different maps with different dimensionality.

\section{Dynamic Self-Organizing Maps (DSOM)}

The proposed algorithm is based on the combination of two neural network models : SOM and Fuzzy ART algorithm. Before presenting details of the proposed DSOM algorithm, a brief overview of the SOM algorithm and Fuzzy ART theory are given.

\subsection{Self-Organizing Maps (SOM)}

The SOM algorithm converts a complex non linear high dimensional input data into low dimension representation using geometric relationships of the input space (Kohonen, 1998).

A typical SOM network consists of two layers neural architecture i.e. input neural layer and output neural layer as given in Fig. 1. Each $p$ dimensional input vector $\mathbf{x}_{k}=\left(x_{k, 1}, x_{k, 2}, \ldots, x_{k, p}\right)^{T}$, in the input layer $\mathbf{X}$ is fully connected to all neurons in the output layer $\mathbf{Y}=\left\{y_{j}: j=1,2, \ldots, m^{2}\right\}$, where $m$ is the order of the neural map in the output layer, which allows the self-organization.

The directed link between the input layer $\mathbf{X}$ to the output layer $\mathbf{Y}$ is given by synaptic weight vector $\mathbf{w}_{j}=\left(w_{j, 1}, w_{j, 2}, \ldots, w_{j, p}\right)^{T} \quad$ (where $j \in\left\{1,2, \ldots, m^{2}\right\}$ is the index of $j^{\text {th }}$ node of the output neuron) from input layer $\mathbf{X}$ to output layer neuron $y_{j}$. These weights (which can be any real number) are updated iteratively by the learning algorithm based on the neighborhood.

The learning principle of the SOM algorithm is to pick an input vector $\mathbf{x}_{k}$ and find the corresponding, so called winner node $y_{j^{*}}\left(j^{*}\right.$ is the index of the winning neuron), by finding the index of the nearest weight vector with $j^{*}=\operatorname{argmin}_{j}\left\|\mathbf{w}_{j}-\mathbf{x}_{k}\right\|$.

Afterwords, the winner node $y_{j^{*}}$ is promoted by adjusting its corresponding weights $\mathbf{w}_{j^{*}}$ towards the nearest input vector $\mathbf{x}_{k}$. In order to ensure that vectors close in distance and topology in the input space are associated with nearby neurons on the map, not only $\mathbf{w}_{j^{*}}$ gets adjusted but also the weights of all nodes in the neighborhood of $y_{j^{*}}$ are also adjusted. The weight vector adjustment is done by the following equation:

$\mathbf{w}_{j}(t+1)=\mathbf{w}_{j}(t)+\alpha(t) \cdot V\left(j, j^{*}, t\right) \cdot\left[\mathbf{x}_{k}-\mathbf{w}_{j}(t)\right]$

Where $t$ represents the time-step and $\alpha(t)$ is learning rate, it is a decreasing function given by:

$\alpha(t)=\alpha_{0}(1-t / T)$

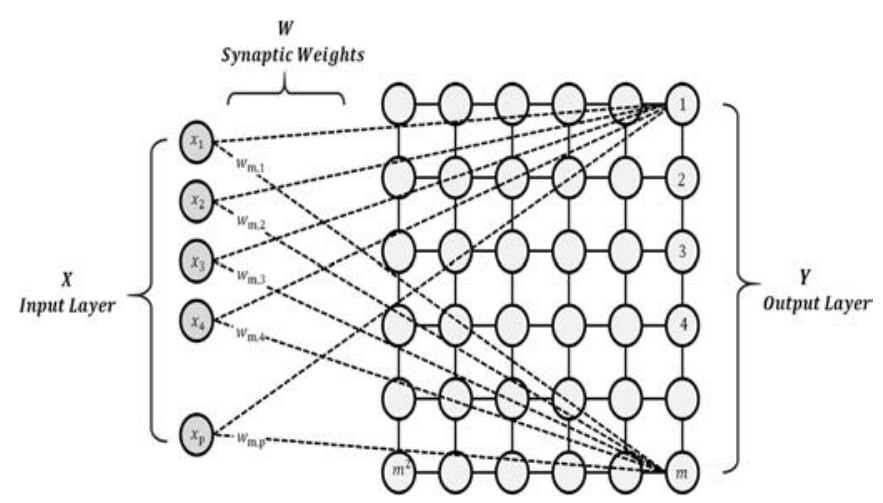

Fig. 1. Schematic SOM network. 
where $T$ is the number of iterations of learning process and $\alpha_{0}$ must be in $[0,1] . V\left(j, j^{*}, t\right)$ is neighborhood function, it represents the influence in term of distance between the winner node $y_{j^{*}}$ and its neighbors $y_{j}$ during the learning process. $V\left(j, j^{*}, t\right)$ is given by:

$V\left(j, j^{*}, t\right)=\exp \left[-\frac{d^{2}}{2 \sigma^{2}(t)}\right]$

where $d^{2}$ is the Euclidean distance between the winner neuron and its neighbors and $\sigma(t)$ is the width of the neighborhood function calculated by:

$\sigma(t)=\sigma_{\max }\left[\sigma_{\max } / \sigma_{\min }\right]^{t / T}$

where $\sigma_{\max }$ is equal to (number of neurones/2), $\sigma_{\min }$ equal to $1 / 2$.

\subsection{Fuzzy ART algorithm}

Fuzzy ART algorithm (Carpenter and Grossberg, 1988) is an unsupervised learning algorithm based on ART theory introduced by Grossberg in 1976 and Fuzzy logic developed by (Zadeh, 1965). It is a neural network model which is capable of rapid learning, recognition and establishment of categories (classes) in response to arbitrary inputs. A typical fuzzy ART network consists of three layers; the first layer is only used to represent the input data, the second layer called matching layer consists of competition process to select the winner neuron with the largest response and the third layer contains selected neurons which represent different categories or clusters. The inputs are matched to these categories using vigilance threshold. If the input and the given category acceptably matches then the given category is chosen and search process ends. If the input is different from the given category (less then the vigilance parameter threshold), the wining neuron is inhibited and new process of search is initialized among the remaining neurons of the second layer. The Fuzzy ART algorithm is given by the following steps for all input samples data presented to the network.

Step 1. Moore (Moore, 1989) described a category proliferation problem that can occur in some Fuzzy ART systems when the input data are not normalized. Normalization of the $p$ dimensional inputs vector $\mathbf{a}_{k}=\left(a_{k, 1}, a_{k, 2}, \ldots, a_{k, p}\right)^{T},\left(\mathbf{a}_{k, i} \in[0,1]\right)$ is achieved by processing each incoming vector $\mathbf{a}_{k}$ as follows:

$\mathbf{x}_{k}=\mathbf{a}_{k}\left|\mathbf{a}_{k}\right|, \quad k=1,2, \ldots, N$

Step 2. Compute the choice function $T_{j}\left(\mathbf{x}_{k}\right)$ for each input vector $\mathbf{x}_{k}$ and weight $\mathbf{w}_{j}$ using:

$T_{j}\left(\mathbf{x}_{k}\right)=\left\|\mathbf{x}_{k} \wedge \mathbf{w}_{j}\right\| /\left(\left\|\mathbf{w}_{j}\right\|+\beta\right), \quad j=1,2, \ldots, m^{2}$

where $\wedge:$ is the Fuzzy AND operator (Zadeh, 1965) defined by: $(\mathbf{a} \wedge \mathbf{b})_{i}=\min \left\{a_{i}, b_{i}\right\}$; the norm $\|$. $\|$ of a given $n$ dimensional vector $\mathbf{v}$ is defined by: $\|\mathbf{v}\|=\sqrt{\sum_{i=1}^{n}\left|v_{i}\right|} ; \mathbf{w}_{j}$ : is the weight vector that connects the input layer with the output layer (neural map network), and $\beta$ : the bias defined in ART algorithm, this value must be within the range $[0,1]$, although values very close to zero are best (Carpenter and Grossberg, 1988).

Step 3. The winner neuron is selected from the activated neurons function $T_{j}\left(\mathbf{x}_{k}\right)$ using the following equation:

$j^{*}=\arg \max _{j}\left(T_{j}\left(\mathbf{x}_{k}\right)\right), \quad j=1,2, \ldots, m^{2}$

Step 4. Determine if resonance occurs by checking if the winner node meets the vigilance threshold $\rho \in[0,1]$, which controls the number of categories (clusters), a high value of $\rho$ gives higher number of clusters and inversely a low vigilance value minimizes category proliferation, i. e: If,

$\frac{\left\|\mathbf{x}_{k} \wedge \mathbf{w}_{j^{*}}\right\|}{\left\|\mathbf{x}_{k}\right\|} \geq \rho$

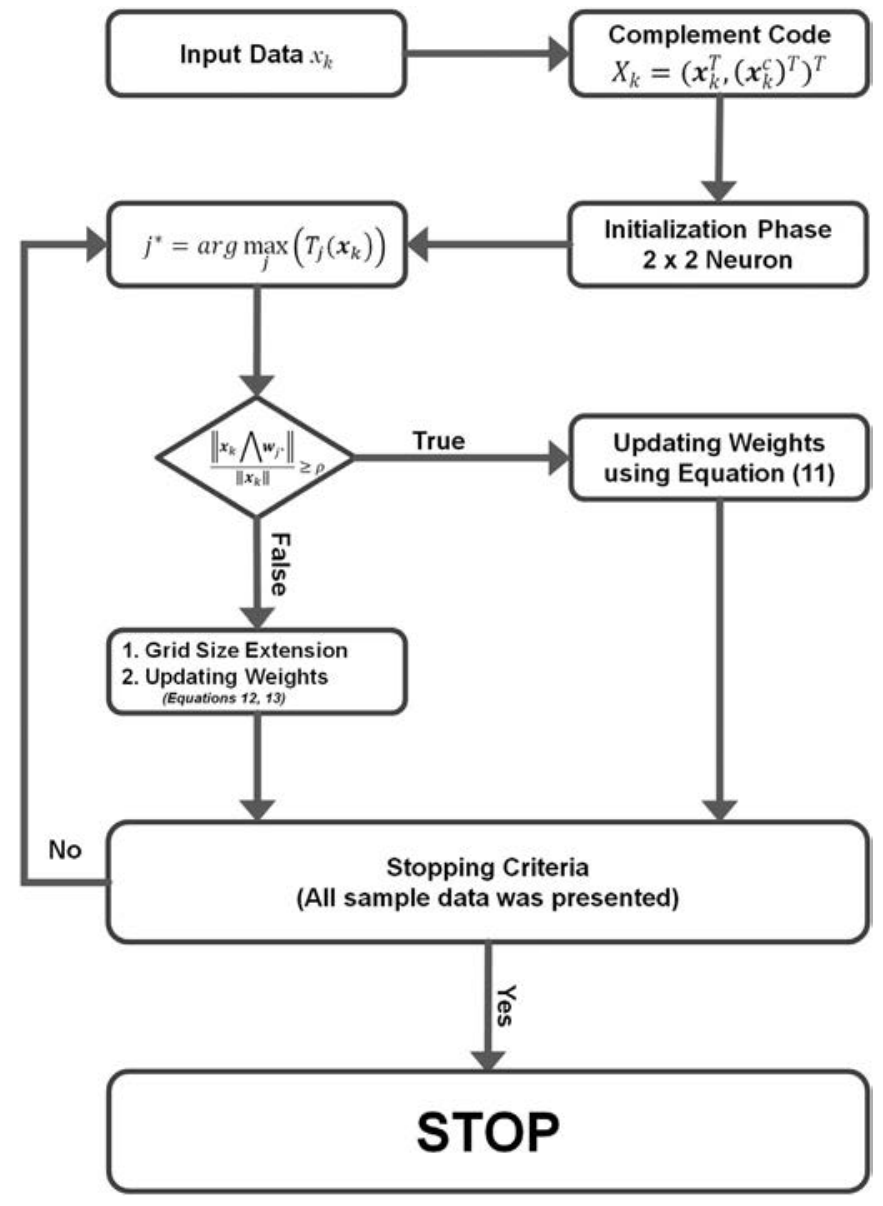

Fig. 2. Synoptic of DSOM algorithm process.

update the weights using:

$\mathbf{w}_{j^{*}}(t+1)=\gamma\left(\mathbf{x}_{k} \wedge \mathbf{w}_{j^{*}}(t)\right)+(1-\gamma) \mathbf{w}_{j^{*}}(t)$

where $\gamma \in[0,1]$ is learning parameter.

Else, set the value of $T_{j}\left(\mathbf{x}_{k}\right)$ to 0 .

Step 5. Repeat steps 3 and 4 until a chosen node meets the vigilance threshold.

\subsection{The proposed DSOM algorithm}

The proposed DSOM algorithm is based on four major steps given below and presented in the Fig. 2:

\section{Data processing}

The input data is normalized using complement coding, which preserve amplitude information by representing both the input data and its complement. The complement of vector $\mathbf{x}_{k}$ denoted by $\mathbf{x}_{k}^{c}$ is given by:

$\mathbf{x}_{k}^{c}=1-\mathbf{x}_{k}$

By applying complement coding to the input vector $\mathbf{x}_{k}$ of $p$ dimensions result in to a $2 p$ dimensional vector given by: $\mathbf{X}_{k}=\left(\mathbf{x}_{k}^{T},\left(\mathbf{x}_{k}^{c}\right)^{T}\right)^{T}=\left(x_{k, 1}, x_{k, 2}, \ldots, x_{k, p}, x_{k, 1}^{c}, x_{k, 2}^{c}, \ldots, x_{k, p}^{c}\right)^{T}$.

2. Initialization phase

The network is initialized with four nodes (a grid of $2 \times 2$ ) with random values from the input vector space. The choice of the number of nodes to initialize the network is justified to implement a 2D lattice structure.

3. Growing phase 


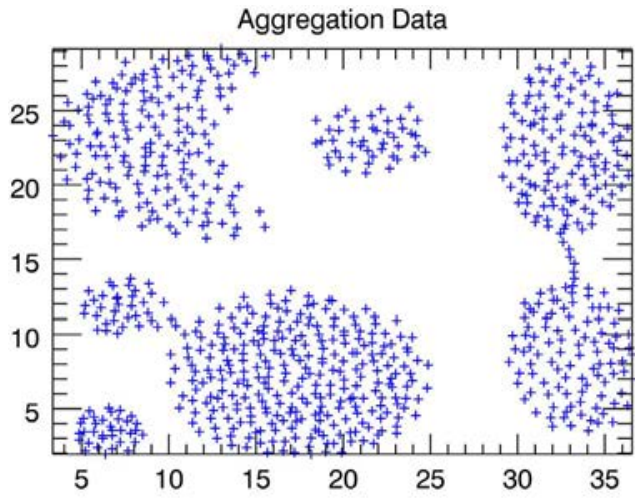

(a)

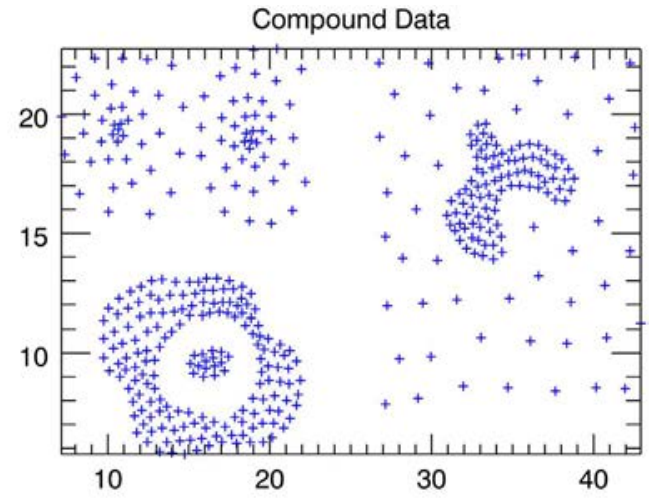

(b)

Fig. 3. Datasets used for experiment. (a) Aggregation dataset, (b) Compound dataset.

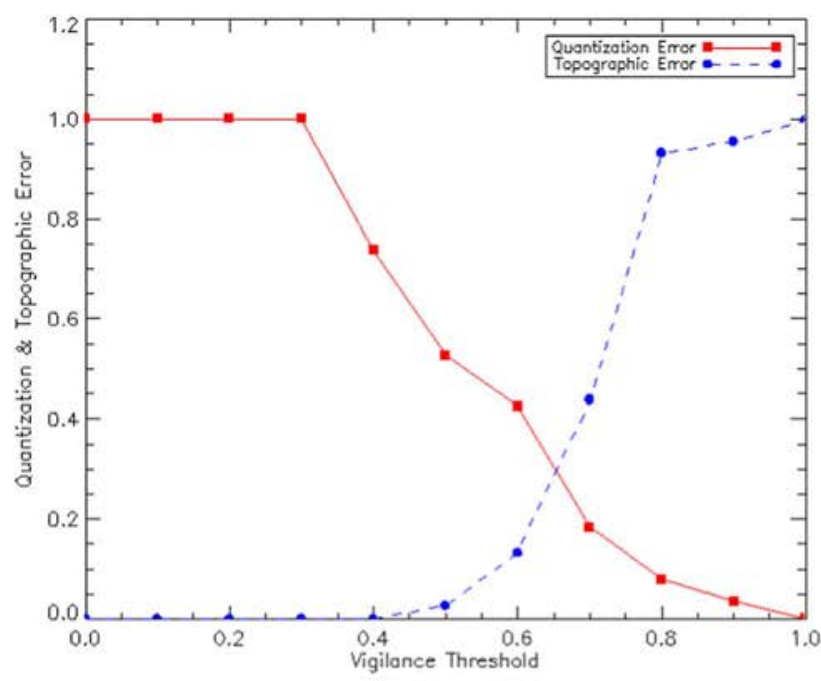

(a)

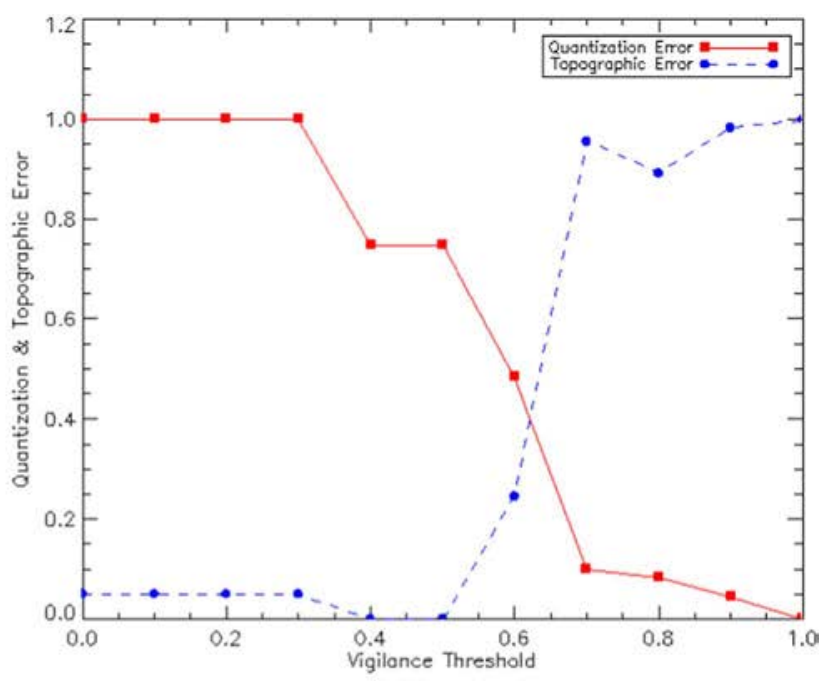

(b)

Fig. 4. Threshold vigilance parameter versus Quantization Error (QE) and Alpha Topographic Error $\left(T E_{\alpha}\right)$.

This is the major step of the proposed DSOM algorithm. The growing process is first based on Eqs. (6), (7) and (8) described in the steps 2-4 of the Fuzzy ART Algorithm.

If the inequality in the Eq. (8) is satisfied then the grid map is not extended, but updating of the weights vectors is computed using the Eq. (11) described in the SOM algorithm.

$\mathbf{w}_{j}(t+1)=\mathbf{w}_{j}(t)+\alpha(t) \times V\left(j, j^{*}, t\right) \times\left(\mathbf{X}_{k} \wedge \mathbf{w}_{j}(t)\right)$

It must be noted that the size of weight vector $\mathbf{w}_{j}$ is extended to $2 p$ dimensions in accordance to the size of $\mathbf{X}_{k}$. Similarly, if the inequality in the Eq. (8) is unsatisfied, new nodes are added to the grid map. In order to keep a square grid, a row and a column of neurons are added and the new weights are computed using Eq. (12) for the row added neurons and Eq. (13) for the column added neurons, respectively.

$\mathbf{w}\left(i_{\text {add }}, j\right)=\frac{1}{2}\left[\mathbf{w}\left(i_{\text {add }}-1, j\right)+\mathbf{w}\left(i_{\text {add }}-2, j\right)\right]$

$\mathbf{w}\left(i, j_{\text {add }}\right)=\frac{1}{2}\left[\mathbf{w}\left(i, j_{\text {add }}-1\right)+\mathbf{w}\left(i, j_{\text {add }}-2\right)\right]$, where $i_{\text {add }}$ and $j_{\text {add }}$ denote the updated number of rows and columns. 4. Stopping Process

The stopping process occurs if all the sample data inputs are presented to the network and the maximum number of iterations is reached.

\subsection{Quality assessment of DSOM based mapping}

Several measures have been used to evaluate the quality of the SOM algorithm and can be extended to study the mapping quality of the DSOM algorithm. Typically two evaluation criteria are used : Quantization and Topographic Errors (Kiviluoto, 1996; Uriarte and Martin, 2005). The Quantization Error (QE) measures the average distance between each input data and the weight of its winner neuron also called best matching unit (BMU). The QE evaluate the fitting of the neural map to the input data space. The smaller the quantization error, the smaller the average of the distance from vector data to the neural map. The QE used in this work is calculated using:

$Q E=\frac{1}{N} \sum_{k=1}^{N}\left\|\mathbf{x}_{k}-\mathbf{w}_{j^{*}}\right\|, \quad \mathrm{j}=1,2, \ldots, \mathrm{N}$ 


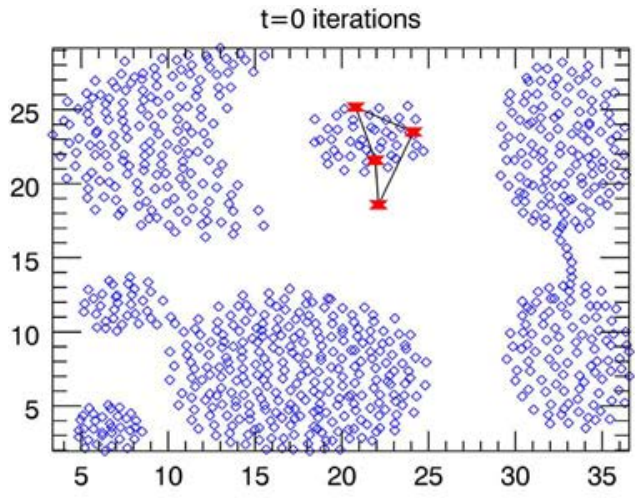

(a)

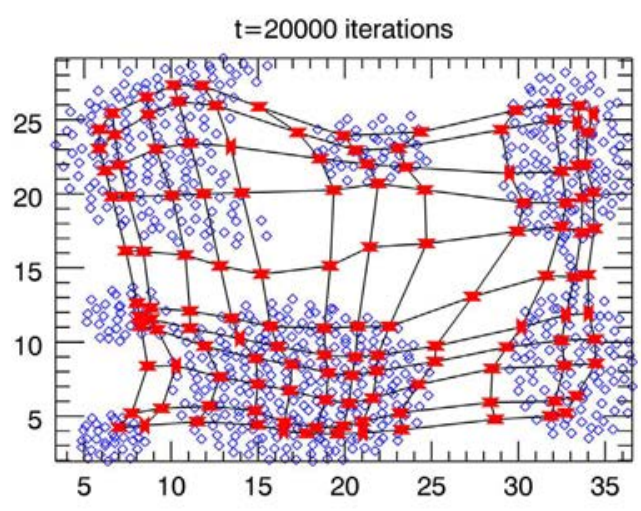

(c)

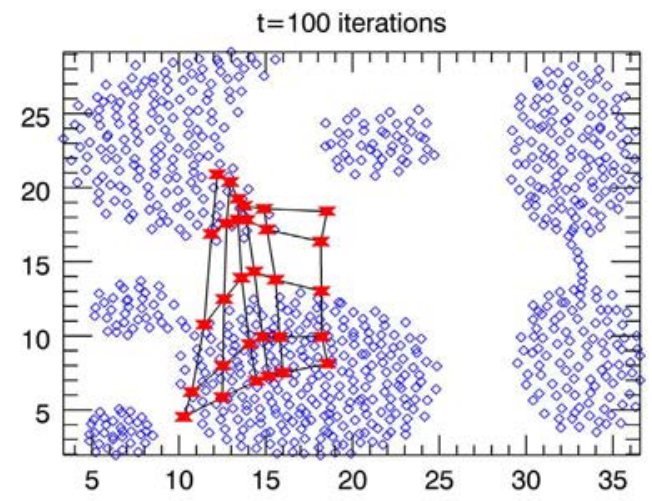

(b)

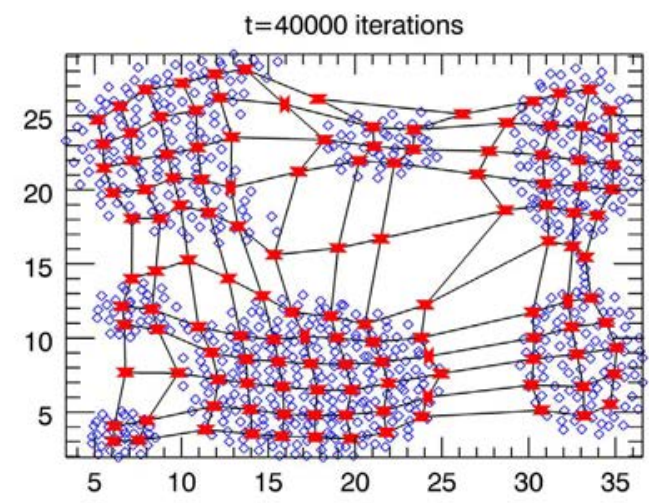

(d)

Fig. 5. Dynamical clustering using DSOM algorithm of Aggregation dataset for iterations: $(t=0$ (Random grid initialization $), t=100, t=20,000$ and $t=40,000)$.

where $N$ is the number of data vectors and $\mathbf{w}_{j}^{*}$ is the BMU.

The second quality measure i.e. the Topographic Error(TE), describes the topological order of the neural grid and measures how continuous mapping from the input space to the grid neural map. This error measures the proportion of all data vectors for which first and second BMUs are not adjacent vectors. Thus, lower the topographic error is, better the SOM preserve the topology. Different Topographic Error(TE) methods are proposed in the literature (Kohonen, 1982; Bauer and Pawelzik, 1992; Bezdek and Pal, 1995), the classical TE measure is given by:

$T E=\frac{1}{N} \sum_{k=1}^{N} U\left(\mathbf{x}_{k}\right)$

$U\left(\mathbf{x}_{k}\right)= \begin{cases}1 & \text { if } 1^{s t} \text { and } 2^{\text {nd }} \text { BMUs are not adjacent } \\ 0 & \text { otherwise }\end{cases}$

In the case of a rectangular lattice the BMU has only four neighbors and the error de-evaluate rectangular maps (Uriarte and Martin, 2005). In addition, it has been observed that in many cases nearby diagonal neurons are the reason why error increases in rectangular maps. This happens because diagonal units represent nearby data although they are not neighbors. Consequently, (Uriarte and Martin, 2005) suggest a new measure for rectangular maps to improve the deficiency of the topographic error. This topographic error called Alfa Topographic Error $\left(T E_{\alpha}\right)$ takes into account different kind of new neighbors. The
$T E_{\alpha}$ is based on assigning weights to different kind of new neighbors as given in Eq. (16), where $Z \neq 0$ is the weight of diagonal neighbor. In the proposed procedure, the rectangular lattice of neural network is adopted. The value of $Z$ is chosen equal to $\sqrt{2}$. This choice is justified by the distance of the BMU to the diagonal neighbor. The case of $Z=1$ means that the first and second BMUs are not adjacent neither diagonals neighbors.

$T E_{\alpha}=\frac{1}{N} \sum_{k=1}^{N} \alpha\left(\mathbf{x}_{k}\right)$

$\alpha\left(\mathbf{x}_{k}\right)= \begin{cases}1 & \text { if } 1^{s t} \text { and } 2^{n d} \text { BMUs are not adjacent } \\ 1 / Z & \text { if } 1^{\text {st }} \text { and } 2^{\text {nd }} \text { BMUs are diagonals } \\ 0 & \text { otherwise }\end{cases}$

The growing process of the DSOM algorithm is controlled by the vigilance parameter given in Eq. (8). For the vigilance parameter a fixed value is used, that empirically minimize the $Q E$ and the $T E_{\alpha}$.

\section{Experimental results}

The objective of a clustering algorithm is to discover grouping of structures inherent in the data. In this section, experimental tests are performed to show the capability of DSOM algorithm for discovering incremental clusters. Two types of experiments are conducted, the first one is the application of the DSOM algorithm for clustering of two 


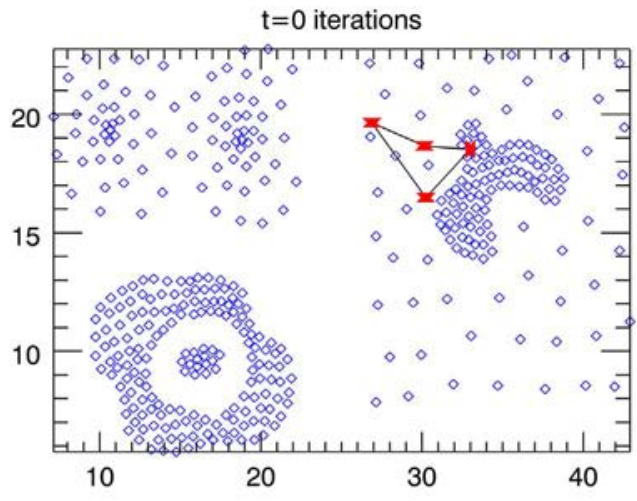

(a)

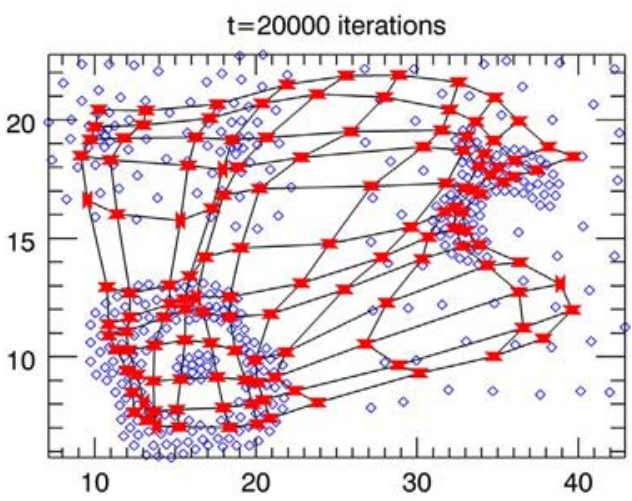

(c)

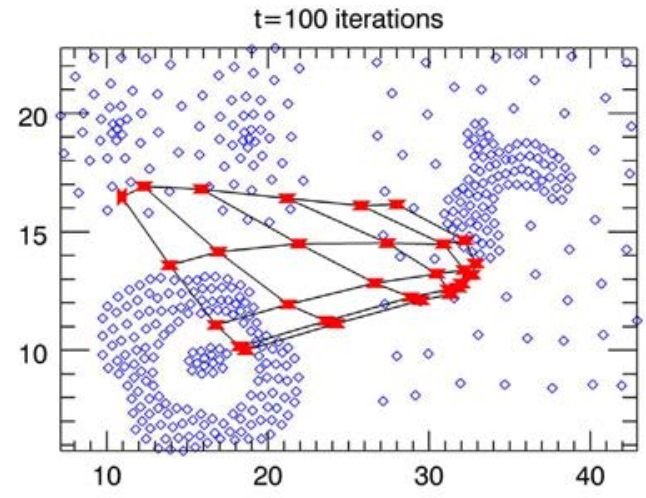

(b)

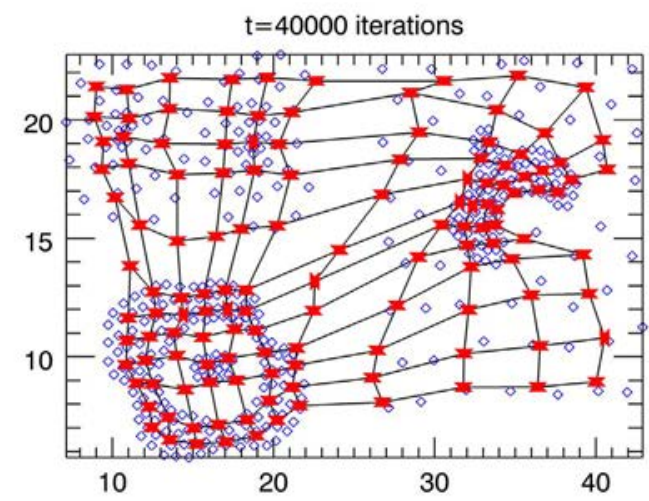

(d)

Fig. 6. Dynamical clustering using DSOM algorithm of Compound dataset for iterations: $(t=0$ (Random grid initialization), $t=100, t=20,000$ and $t=40,000)$.

types of synthetic datasets. The second experiment demonstrates the application of the proposed DSOM algorithm on real sonar images.

\subsection{Application to synthetic data}

To establish the importance and applicability of the proposed DSOM, data-sets containing features and characteristics which are known to create difficulties and complications are used for clustering in real scenarios such as uneven size and shape of clusters, clusters with slender links, etc. The data used for experiment are two datasets shown in Fig. 3. The dataset in the Fig. 3(a), called Aggregation data used in (Gionis et al., 2007), contains 7 clusters, 788 vectors in 2-dimensions. The second one is Compound data shown in Fig. 3(b) and contains 399 vectors, in 2-dimensions with 6 clusters used by (Zahn, 2007). These are benchmark datasets used in standard clustering algorithms for testing the performance and accuracy of the clustering algorithms.

The first step in the test consist of the choice of the vigilance parameter $\rho$. This parameter is very important for the growing process step. It allows to control the number of nodes in the neural grid, whereas a high value gives a higher number of neurones and inversely low vigilance values minimize neurones proliferation. The quantization and the topographic errors are used to select an optimal value of vigilance parameter. The optimal value is the one that minimizes both topographic and quantization measures. Fig. 4 represent the graphs of the quantization and topographic errors of DSOM algorithm applied on Aggregation and Compound datasets for different values of $\rho$ between $[0,1]$.
In Fig. 4, we can see for both Aggregation and Compound datasets, the $\mathrm{QE}$ is equal to 1 for values of $\rho$ between $[0,0.3]$. In this case, the size of the neural grid is the same as that of the initialized grid $2 \times 2$ (no expansion of the grid). But for the values of $\rho$ between [0.4, 1], the $Q E$ decreases and the grid size is incrementally extended. On the other side, the behavior of the $T E_{\alpha}$ error is inverse to that of the $Q E$ error. For values of $\rho$ in $[0,0.3]$ the $T E_{\alpha}$ is constant and increases for values of $\rho$ between $[0.4,1]$. The intersection of the two measures $\left(\mathrm{QE}\right.$ and $\left.T E_{\alpha}\right)$ give the optimal value of the vigilance parameter $\rho$. From the Fig. 4 , the optimal values of vigilance parameter for Aggregation dataset is $\rho=0.65$ and $\rho=0.62$ for Compound dataset.

In the Fig. 5 and 6, the results of the application of DSOM algorithm to the synthetic datasets Aggregation and Compound are presented. The map grid is shown for different time step iterations, $t=0$ represent the initialization of the DSOM algorithm with random map grid of $2 \times 2$. Similarly, for iteration ( $t$ ) equal to $(100,20,000$ and 40,000$)$ the map grid size is extended and adapts its geometric form to the topological form of the input data. For example, in the case of the application of DSOM algorithm to Aggregation data (see Fig. 5), the neural grid is initialized by a neural map size of $2 \times 2$ neurons at $t=0$, then dynamically the size of the map is growing. The map size at $\mathrm{t}=100$ is $5 \times 6$ neurons and the final grid map size grid $11 \times 12$ at $\mathrm{t}=40,000$.

\subsection{Application to sidescan sonar images}

In these experiment dataset of real sonar images are used to asses the capabilities of the proposed algorithm in creating clusters. 

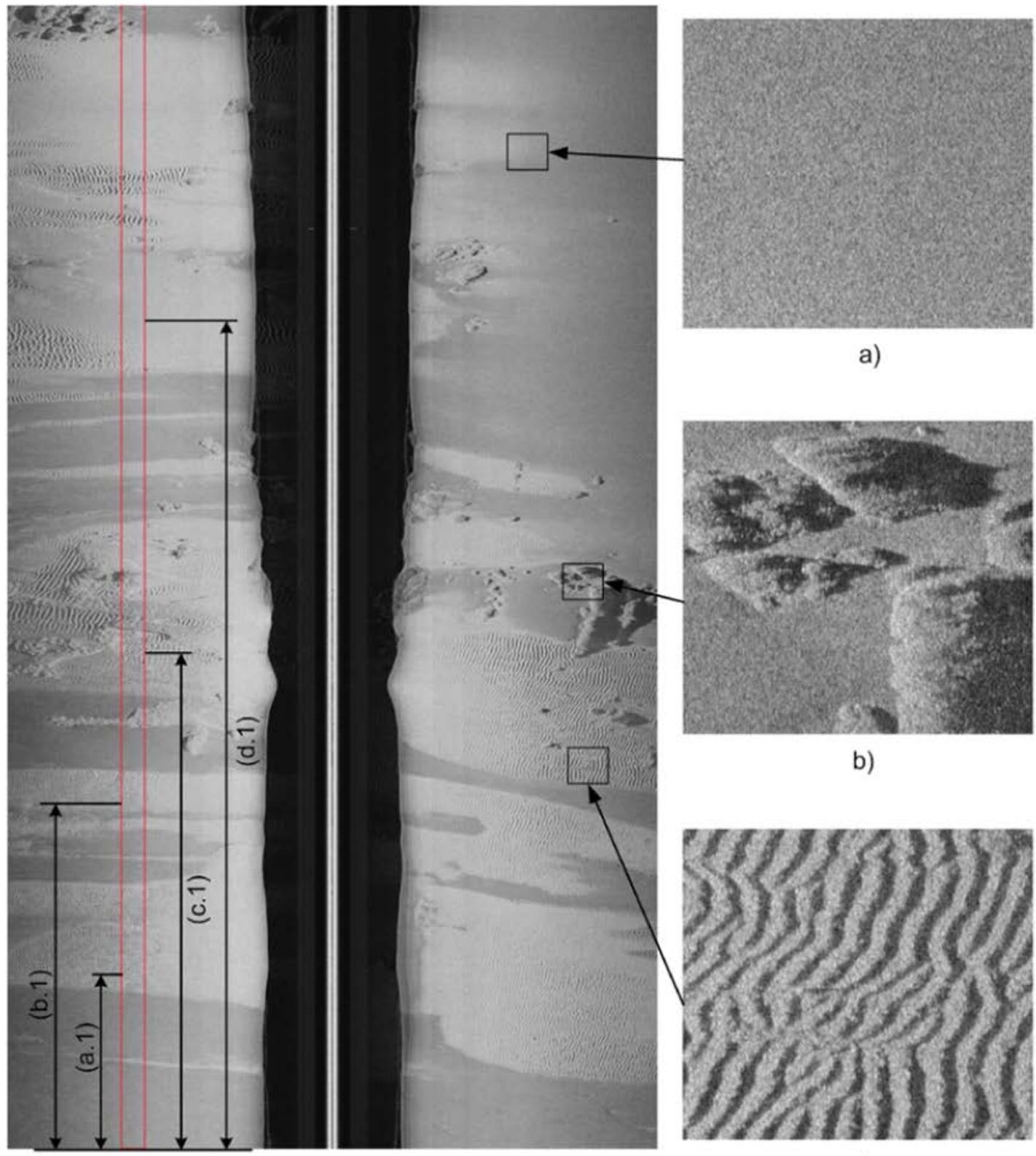

a)

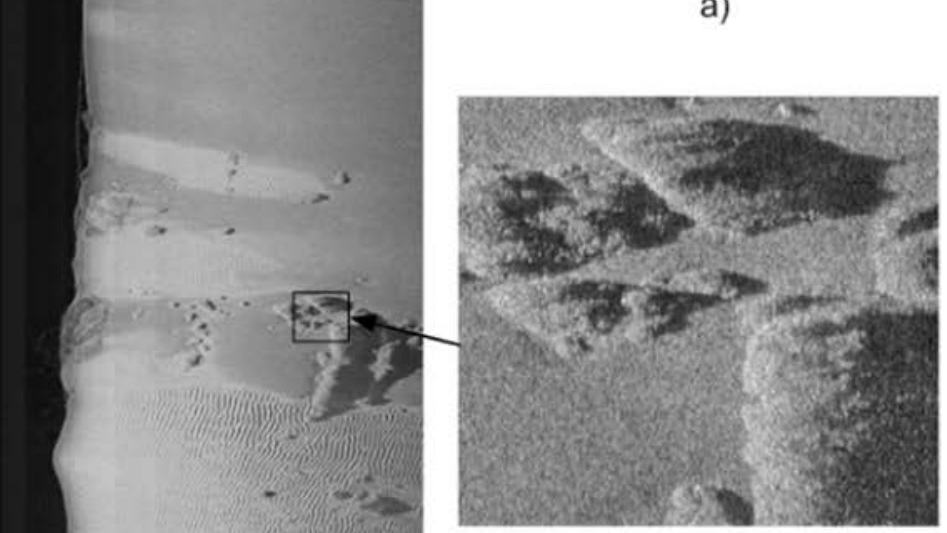

b)

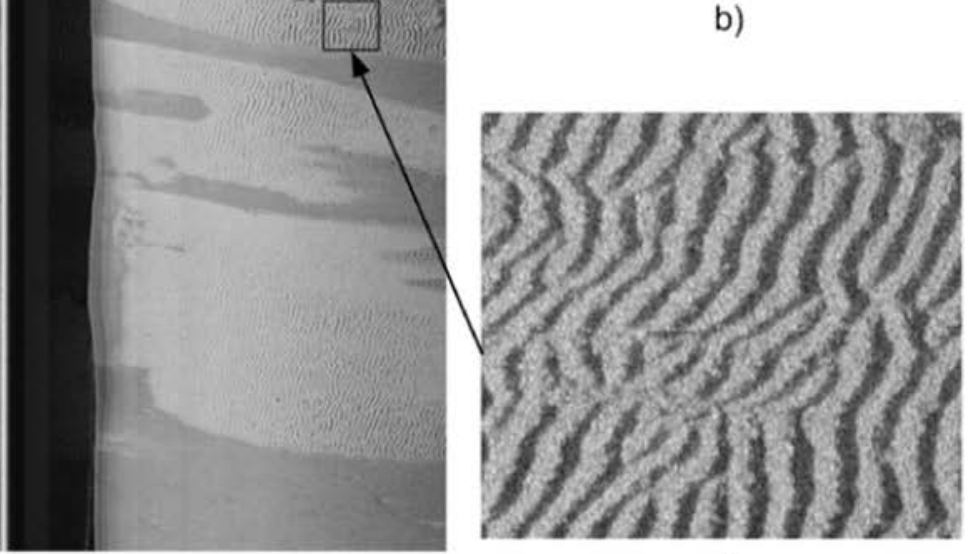

c)

Fig. 7. Example of sonar image acquired by Klein 5000 sidescan sonar system and three types of seabed: a) Silt, b) Rock, c) Ripples.

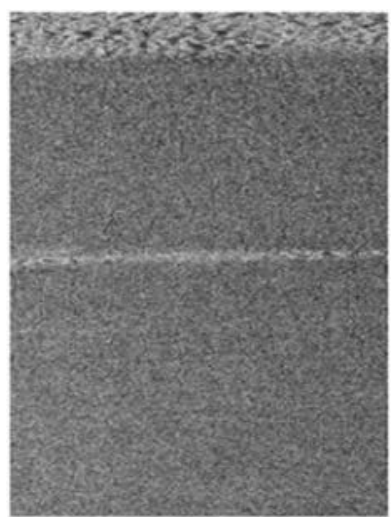

(a)

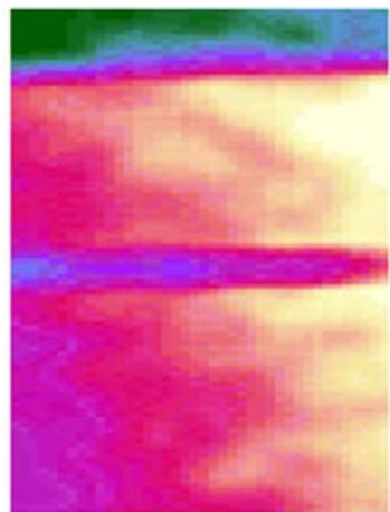

(b)

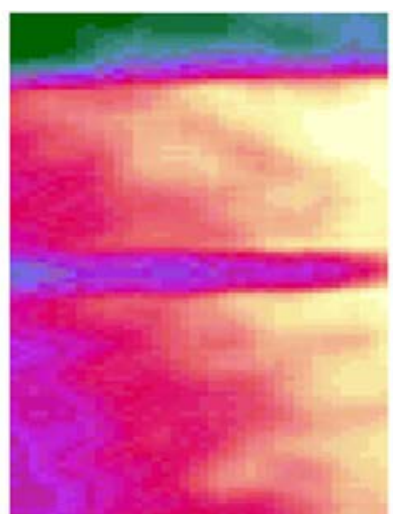

(c)

Fig. 8. a) Sonar image obtained from Fig. 7, (Fig. 1), b) Classification results using DSOM, c) Classification results using classical SOM with neural map of $10 \times 10$ 


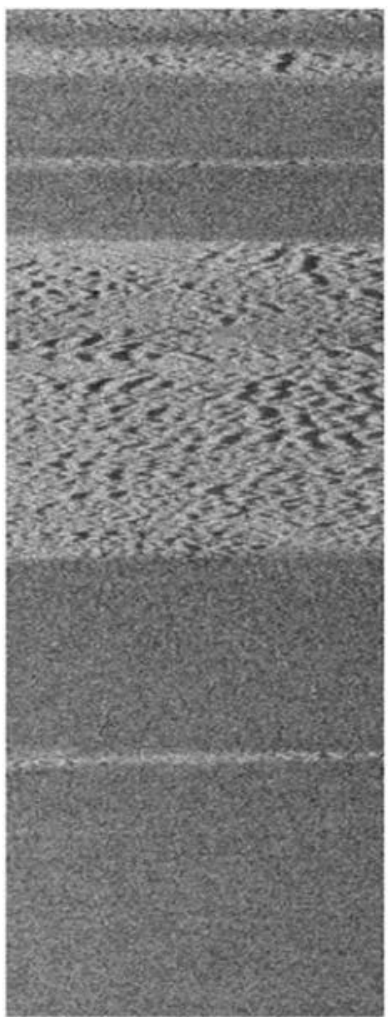

(a)

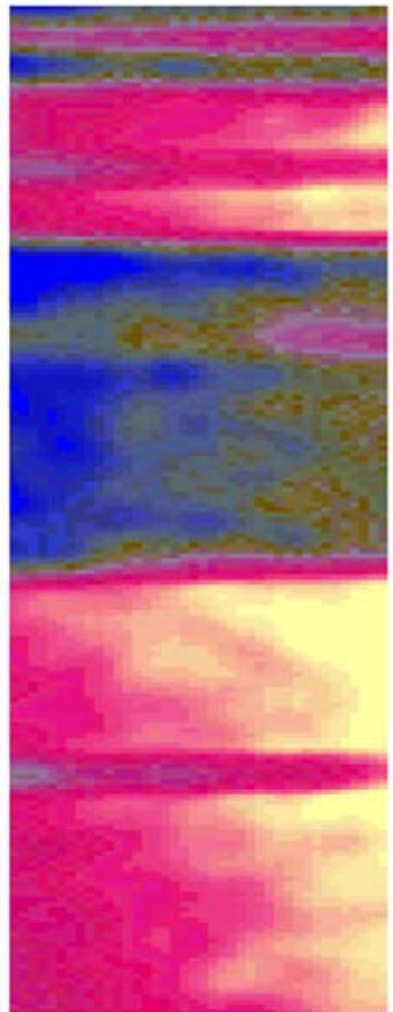

(b)

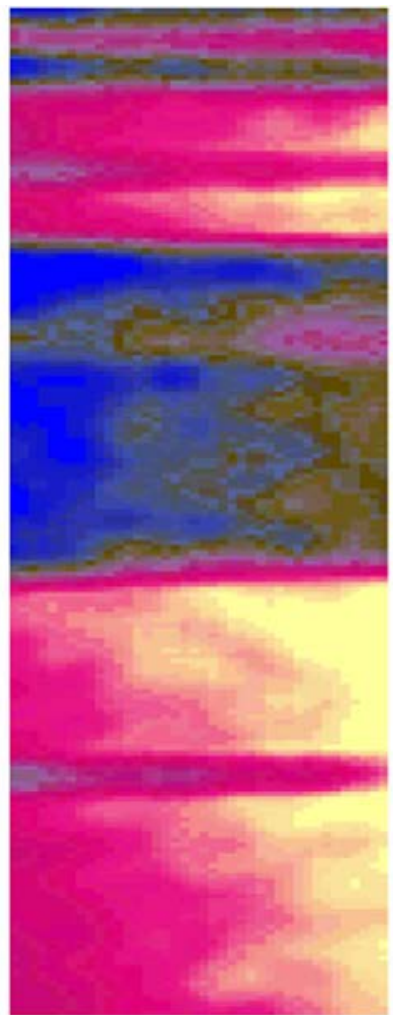

(c)

Fig. 9. a) Sonar image obtained from Fig. 7, (Fig. 1), b) Classification results using DSOM, c) Classification results using classical SOM with neural map of $10 \times 10$.

\subsubsection{Sonar data presentation}

The data used is obtained during the BP02 (Battle- space Preparation) experiments carried out by the SACLANT Undersea Research Center in La Spezia, Italy. The system used is the Klein 5000 sidescan sonar operating at $455 \mathrm{kHz}$. The sensor can work on two modes of resolution (low and high). In low resolution mode, the alongtrack resolution is $20 \mathrm{~cm}$ and the maximum range is $150 \mathrm{~m}$ on each side of sonar which gives a swath of $300 \mathrm{~m}$. In high resolution mode, the along-track resolution is $10 \mathrm{~cm}$ and the maximum range is limited to $75 \mathrm{~m}$ (i.e. a swath of $150 \mathrm{~m}$ ). In both modes, the across-track resolution is $3 \mathrm{~cm}$. In our case we only use images acquired in high resolution mode.

An example of high resolution sonar image is shown in Fig. 7 with along-track resolution of $10 \mathrm{~cm}$ and across-track resolution of $3 \mathrm{~cm}$, the total size of the image in pixel is $4221 \times 450$ making the total along track distance is approximately $4200 \mathrm{~m}$ or $4.2 \mathrm{~km}$. In this image, different types of seabed can be observed: homogeneous area in the top right of the Fig. 7 is representing silt type sediment. Similarly, sand ripples, rock sediments and other more complex areas can be observed and distinguished.

\subsubsection{Feature extraction}

The proposed approach is tested on sonar images presented in Fig. 7. The conditions of acquiring a sidescan sonar image near the bottom allow the appearance of seabed textures. Several methods of texture analysis are proposed in the literature, in the proposed work, different features computed from texture analysis and spectral analysis of sonar images are used as input vector to DSOM algorithm. The details about the features used for sonar images in this work are given in Nait-Chabane et al. (2013).

The texture analysis of sonar image are based on the Gray Level Cooccurrence Matrix(GLCM). GLCM features are a second-order statistical tool used for texture analysis of images proposed by Haralick
(Haralick et al., 1973). The GLCM of an image is obtained by calculating the number of transitions for each pair of gray level $(\mathrm{i}, \mathrm{j})$ of a given distance $(d)$ and angular direction $(\theta)$. In this work, the following Haralick features are used: Entropy, Contrast, Heterogeneity, Homogeneity, Correlation, Maximum of probability, Kurtosis and Elongation Factor.

The second set of features, extracted from sonar images are based on 2D Fourier analysis. Fourier analysis can be used to study the properties of textured scenes, for example the power spectrum reveals information on the coarseness/fineness (periodicity) and directionality of a texture. Texture directionality is preserved in the power spectrum because it allows directional and non-directional components of the texture to be distinguished. Three features calculated directly from the amplitude spectrum of the 2D Fourier transform are: mean of the Fourier amplitude, variance of the amplitude and power of the amplitude (i.e power spectrum). Three other features are calculated from the power spectrum of 2D Fourier transform. Fourier power spectrum is separated into three spectral bands: low pass, medium pass and high pass frequencies power. The detail about these features can be found in Nait-Chabane et al. (2013).

\subsubsection{Sonar images clustering}

To improve the dynamic learning in case of new seabed, first, an image Fig. 8.a is tested by DSOM algorithm then, new areas of the sonar image in Fig. 9(a), Fig. 10(a) and Fig. 11(a) are gradually added during the application of the DSOM algorithm. The images in [Fig. Fig. 8(a), Fig. 9(a), Fig. 10(a) and Fig. 11(a)] belong to the red rectangular block of Fig. 7 and labeled by $a 0.1, b 0.1, c 0.1$ and $d 0.1$ respectively.

The result of the DSOM algorithm is a discrete map of neurons where each node or neuron has a given position in the map with corresponding weight vector of the same dimension as the features vector. The DSOM algorithm transforms the high dimension feature 


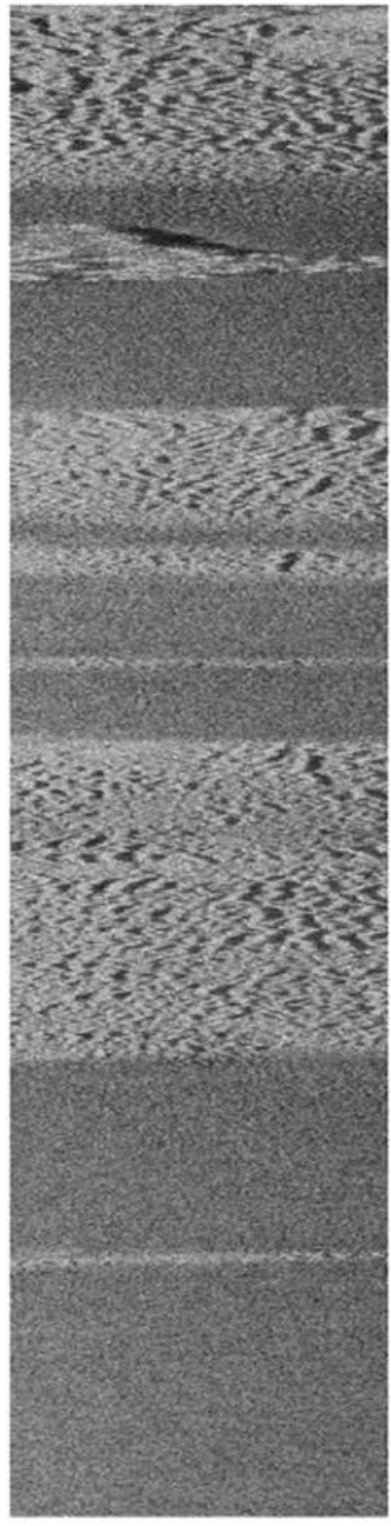

(a)
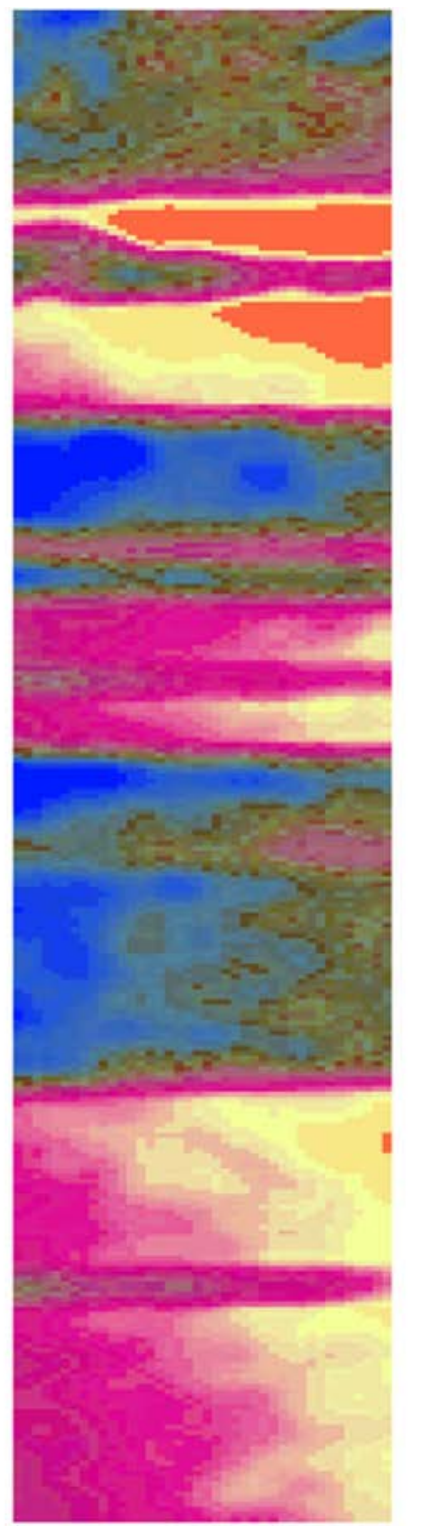

(b)

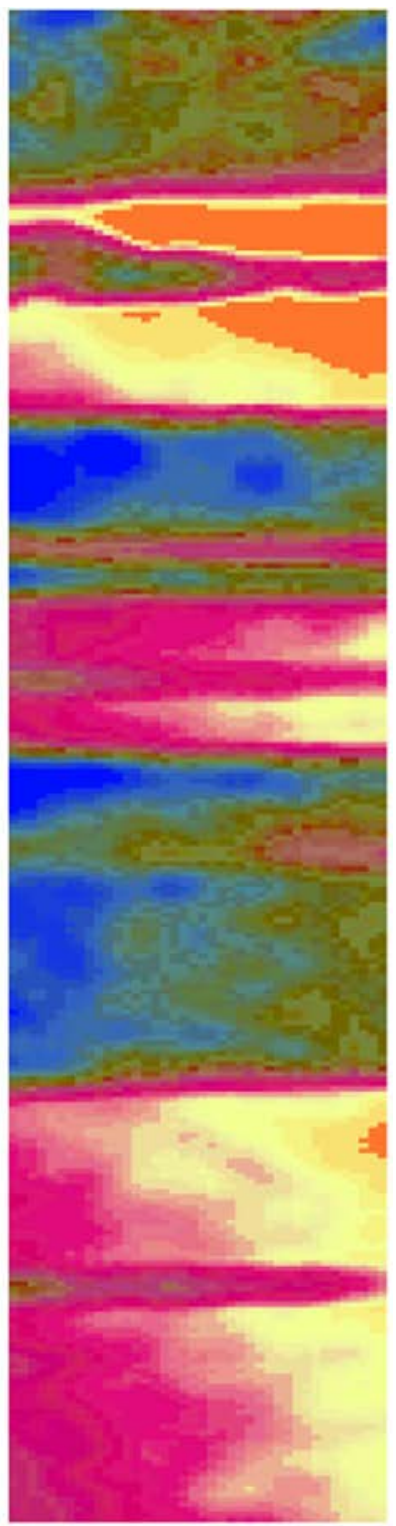

(c)

Fig. 10. a) Sonar image obtained from Fig. 7, (Fig. 1), b) Classification results using DSOM, c) Classification results using classical SOM with neural map of $10 \times 10$.

vector into a two dimensional discrete map of neurons subject to a topological constraint with particularity of neighborhood preservation. The classical approach for color attribution is chosen randomly using three weights (R, G, and B, G, and B). An Euclidean distance is used to attribute the color of each neuron in the map. The neurons that are close in distance will have close colors on the map. To overcome the problem of color attribution and to have a physical link between the color and the seabed type, a solution based on application of Principle Components Analysis (PCA) is used. PCA is applied to reduce the dimensionality of the weight space to only three components. The application of PCA in this case can be seen as a projection of the neuronal map on the input space. This projection allows to have a link between the position of neuron and a given color defined by features vector values. The first three axes of PCA result represent more than of 90 percent of total variance and define the Red, Green and Blue (RGB) color table. More details of the solution presented about the color attribution of neural map is given in (Nait-Chabane and Zerr, 2014).
Fig. 12 show the results of neural maps grid obtained by DSOM algorithm with correspondent color for each neuron. Similarly, Fig. 12(a), (b), (c) and $(d)$ represent the grid map of the application of DSOM algorithm on images in Fig. 8(a), Fig. 9(a), Fig. 10(a) and Fig. 11(a).

Each neuron with its correspondent color represent a given class. Each input data of sonar images is assigned to correspondent neuron according to Euclidean measure.

Fig. 8(b), Fig. 9(b), Fig. 10(b) and Fig. 11(b) present the classification results of the proposed DSOM on Fig. 8(a), Fig. 9(a), Fig. 10(a) and Fig. 11(a). The results give a good classification of sonar seabed and the DSOM algorithm manages the gradual addition of new sonar images area. If the image shown to the DSOM neural map is already seen, the grid size does not change and a color already used for that same seabed is assigned. However, if the new presented area has not been seen by the DSOM, the size of the grid changes and a new color is created for the seabed.

A comparison of sonar images classification is finally made between proposed DSOM algorithm and classical SOM algorithm with a predefined 


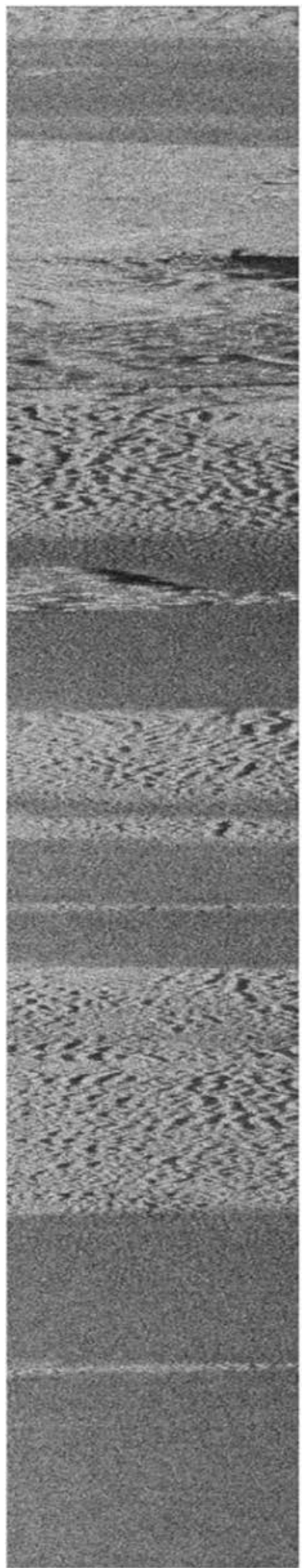

(a)
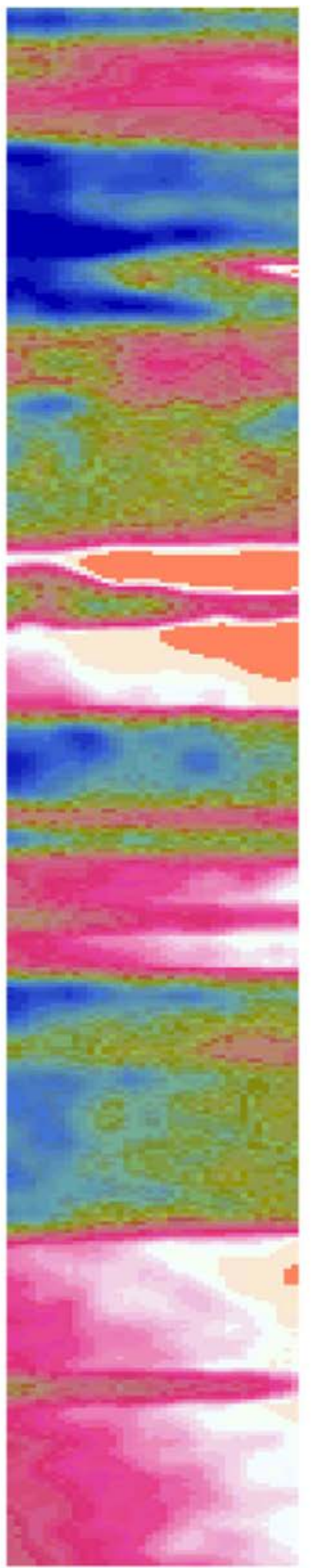

(b)
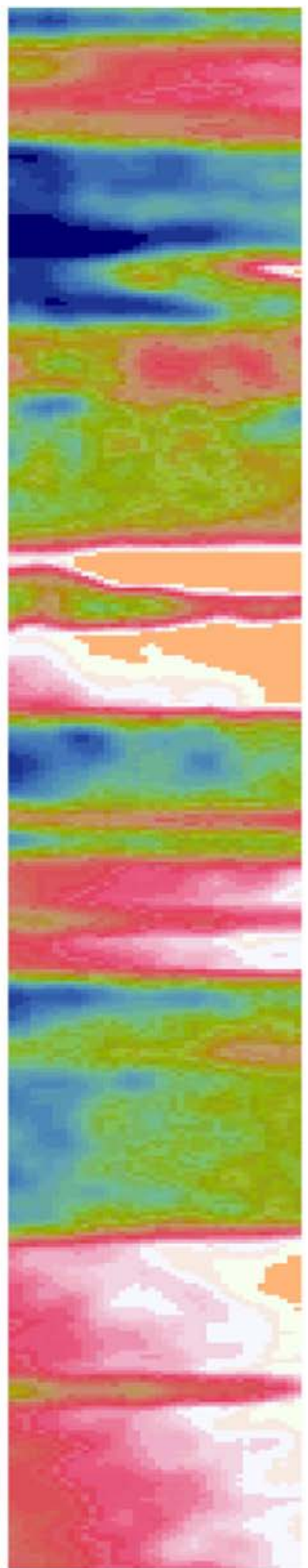

(c)

Fig. 11. a) Sonar image obtained from Fig. 7, (d.1), b) Classification results using DSOM, c) Classification results using classical SOM with neural map of $10 \times 10$.

map size of $10 \times 10$. The classification results of the application of SOM algorithm are given in the Fig. 8(c), Fig. 9(c), Fig. 10(c) and Fig. 11(c). It clearly show that the results obtained by the classical SOM algorithm are similar to those given by a DSOM when SOM is feed with the predefined number of neurons. Despite the initialization of DSOM algorithm with only $2 \times 2$ neurons, the incremental characteristic of the proposed approach allows the detection of new classes (new seabed). The SOM algorithm converges to a DSOM algorithm if the number of neurons (classes) is known to the SOM approach.

Fig. 13 is another example of application of the proposed DSOM 


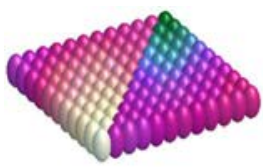

(a)

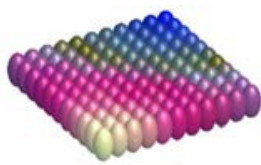

(b)

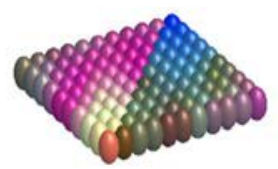

(c)

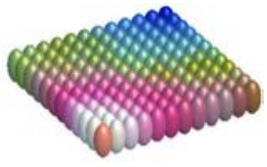

(d)

Fig. 12. Neural map grid obtained using DSOM algorithm on the test images shown in Fig. 8.a, Fig. 9.a, Fig. 10.a and Fig. 11.a.
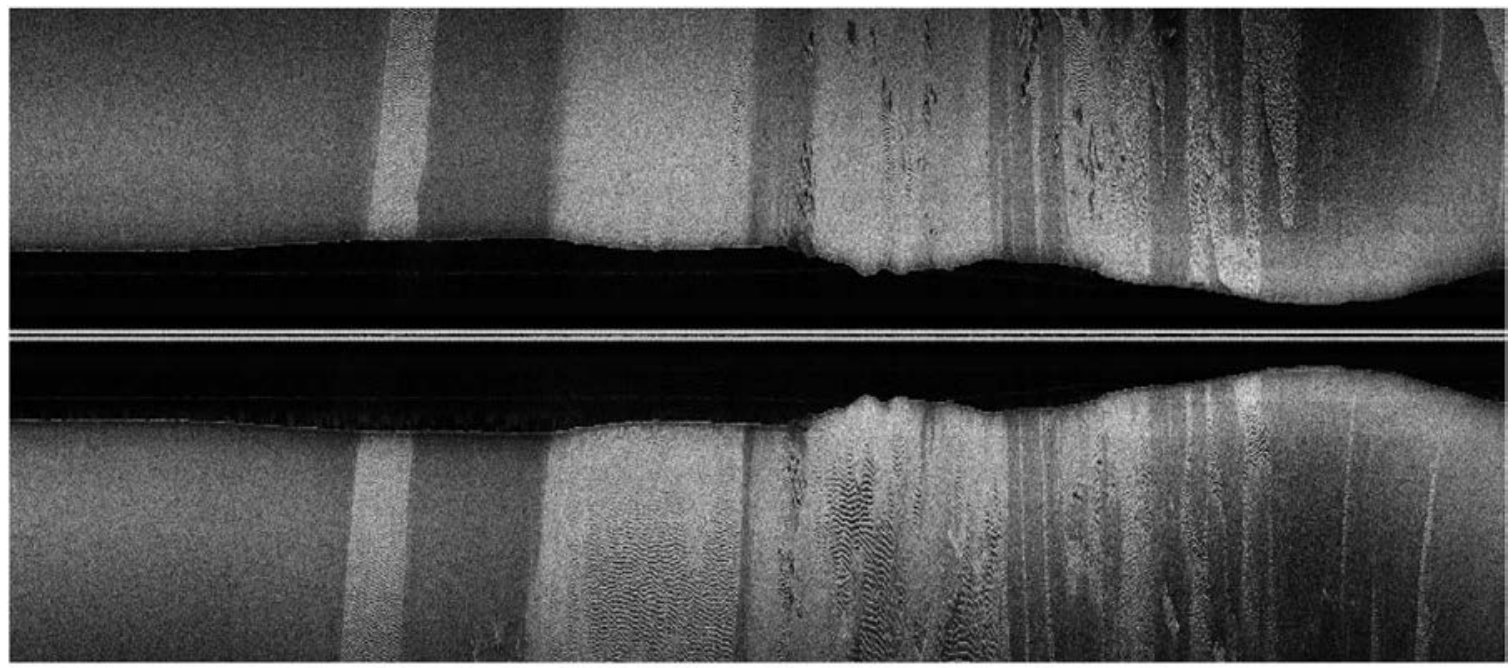

(a)

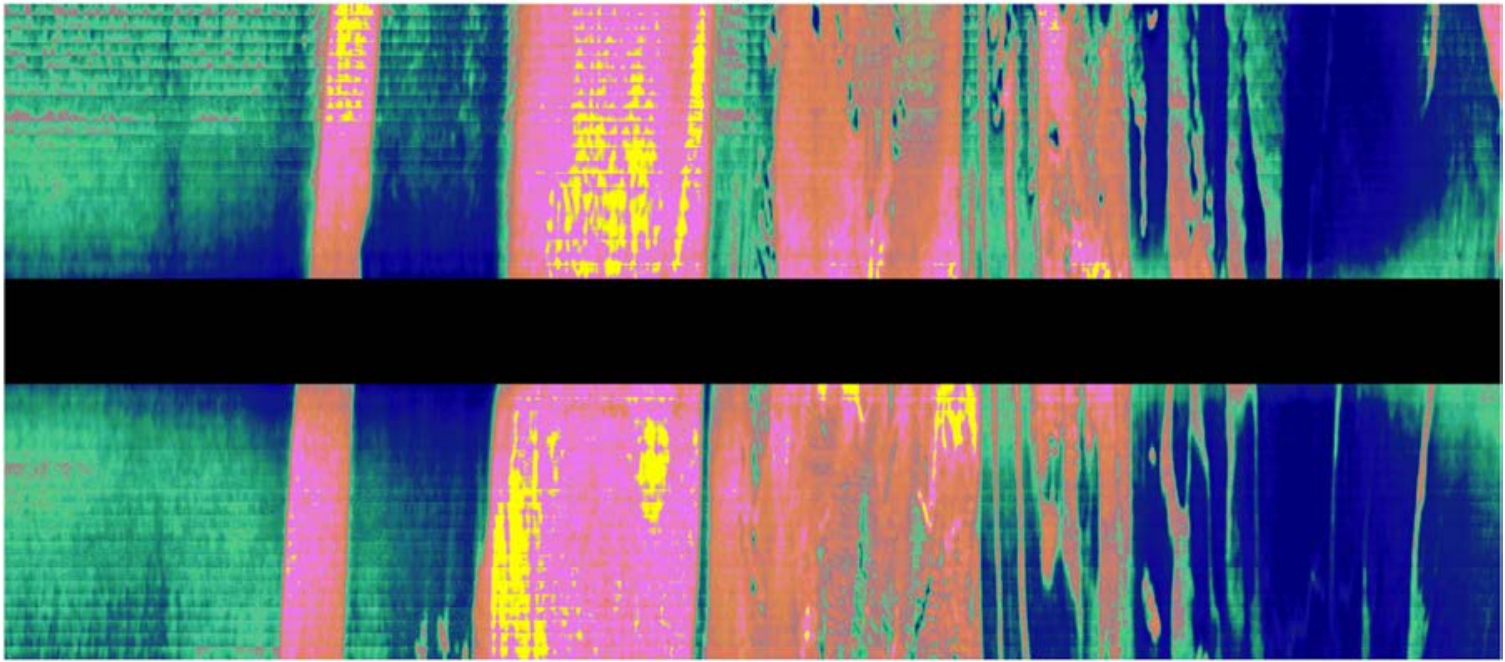

(b)

Fig. 13. a) Sonar Data, b) Proposed DSOM Classification map.

algorithm. Fig. 13.a shows the high resolution sonar image (horizontally placed) acquired using Klein 5000 sidescan sonar operating at $455 \mathrm{kHz}$, with along-track resolution of $10 \mathrm{~cm}$ and across-track resolution of $3 \mathrm{~cm}$. The total size of the image in pixel is $2520 \times 450$, making the total along track distance of around $2520 \mathrm{~m}$ or $2.52 \mathrm{~km}$. Fig. 13.b show the classification map resulted by applying the proposed DSOM algorithm over Fig. 13.a. It can be observed from the resulted grid map in Fig. 13.b that the proposed DSOM has clustered the seabed into different colors. That is, regions with similar features and characteristics are dynamically mapped to one color while regions with different features are mapped to different colors.

\subsection{Computational complexity}

The task of assigning the data to cluster is achieved by finding the distance between the datapoint and the weight of each neuron and by assigning the data neurons whose weight vector is closest. This distance calculation process is performed during the learning process for all the data point and every iteration. The computation for each learning step is order of $O(N)$, where $N$ is the number of candidates for the winner neuron and the overall computational complexity of the proposed DSOM is order of $O(K N)$ as for each learning step to realize adequate numerical precision the quantity of iterations should be at least some multiple of $N$. 


\section{Conclusion and future work}

In this paper, we investigated a new dynamic approach for clusters detection. The proposed approach is based on the combination of SelfOrganizing Maps and Fuzzy ART algorithms. To test the algorithm clustering capabilities, experiments are conducted on simulated data and real sonar data. The results obtained are promising and highlights the capabilities of the proposed DSOM. The distinction and the innovation of the proposed DSOM is based on the construction of dynamic size of neurons that incrementally characterize the detection of new classes systematically, without the need of providing predefined number of classes unlike SOM. As a continuation of the proposed work, in future, the robustness to discover new cluster autonomously in real time and in complex situation will be analyzed, which will ensure the reliability under complex situations and difficult terrain.

\section{Acknowledgments}

The authors would like to thank the Center for Maritime Research and Experimentation (CMRE) and the GESMA (DGA/TN) for allowing the inclusion of data from the BP02 experiment.

\section{References}

Acharyya, R., 2008. A New Approach for Blind Source Separation of Convolutive Sources, VDM Verlagr.

Alahakoon, D., Halgamuge, S., Srinivasan, B., 2000. Dynamic self-organizing maps with controlled growth for knowledge discovery. IEEE Trans. Neural Netw. 11 (3), 601-614.

Bauer, H.-U., Pawelzik, K.R., 1992. Quantifying the neighborhood preservation of selforganizing feature maps. IEEE Trans. Neural Netw. 3 (4), 570-579.

Bezdek, J.C., Pal, N.R., 1995. An index of topological preservation for feature extraction. Pattern Recognit. 28 (3), 381-391.

Bourgeois, B., Walker, C., 1919. Sidescan Sonar Image Interpretation With Neural Networks. In: OCEANS '91. Ocean Technologies and Opportunities in the Pacific for the 90 's. Proceedings, pp. 1687-1694.

Carpenter, G.A., Grossberg, S., 1988. The ART of adaptive pattern recognition by a selforganizing neural network. Computer 21 (3), 77-88.

Carpenter, G.A., Grossberg, S., Lesher, G.W., 1998. The what-and-where filter: a spatial mapping neural network for object recognition and image understanding. Comput. Vision. Image Underst. 69 (1), 1-22.

Duda, R., Hart, P., Stork, D., 2001. Pattern Classification second edn.. Wiley, (ISBN 0471-05669-3).

Fritzke, B., 1994. Growing cell structures a self-organizing network for unsupervised and supervised learning. Neural Netw. 7 (9), 1441-1460.

Gionis, A., Mannila, H., Tsaparas, P., 2007. Clustering aggregation. ACM Trans. Knowl. Discov. Data (TKDD) 1 (1), 1-30.

Hansen, L., Salamon, P., 1990. Neural network ensembles. IEEE Trans. Pattern Anal. Mach. Intell. 12 (10), 993-1001.

Haralick, R.M., Shanmugam, K., Dinstein, I., 1973. Textural Features for Image Classification, IEEE Transactions on Systems, Man, and Cybernetics SMC-3, 6, pp. 610-621, ISSN 0018-9472, http://dx.doi.org/10.1109/TSMC.1973.4309314.

Hastie, T., Tibshirani, R., Friedman, J., 2009. The Elements of Statistical Learning: Data
Mining, Inference, and Prediction. Springer, New York, NY.

Kim, M.-H., Jang, D.-S., Yang, Y.-K., 2001. A robust-invariant pattern recognition model using Fuzzy ART. Pattern Recognit. 34 (8), 1685-1696.

Kinnunen, T., Kamarainen, J.-K., Lensu, L., Unsupervised, H.K., 2012. object discovery via self-organisation. Pattern Recognit. Lett. 33 (16), 2102-2112.

Kiviluoto, K., 1996. Topology preservation in self-organizing maps. In: Neural Networks, 1996, IEEE International Conference on, 1, pp. 294-299. http://dx.doi.org/10. 1109/ICNN.1996.548907.

Kohonen, T., Oja, E., Simula, O., Visa, A., Kangas, J., 1996. Engineering applications of the self-organizing map, Proceedings of the IEEE, 84, 10, pp. 1358-1384.

Kohonen, T., 1982. Self-Organizing Formation of topologically Correct Feature Maps. In: Biological Cybernitics, 46, pp. 59-69.

Kohonen, T., 1998. The self-organizing map. Neurocomputing 21 (1), 1-6.

Kohonen, T., 2013. Essentials of the self-organizing map. Neural Netw. 37 (0), 52-65.

Liu, L., Wang, B., Zhang, L., 2010. An approach based on self-organizing map and fuzzy membership for decomposition of mixed pixels in hyperspectral imagery. Pattern Recognit. Lett. 31 (11), 1388-1395.

Maillard, E., Zerr, B., Merckle, J., 1992. Classification of Texture by an Association Between a Perceptron and a Self-Organizing Feature Map. In: Vandewalle, J., Boite, R., Moonen, M., Oosterlinck, A., (Eds.), EUSIPCO-92, Proceedings of the Sixth European Signal Processing Conference, II, Elsevier, Amsterdam, Netherlands, pp. 1173-1176.

Martinetz, T.M., Berkovich, S.G., Schulten, K.J., 1993. Neural-gas' network for vector quantization and its application to time-series prediction. IEEE Trans. Neural Netw. 4 (4), 558-569.

Moore, B., 1989. ART 1 and pattern clustering. In: Touretzky, D., Hinton, G., Sejnowski, T., (Eds.). In: Proceedings of the 1988 Connectionist Models Sumer School, San Mateo, CA: Morgan Kaufmann Publishers.

Muller, R.D., Overkov, N.C., Royer, J., Dutkiewicz, A., Keene, J.B., 1997. Seabed classification of the South Tasman Rise from SIMRAD EM12 backscatter data using artificial neural networks. Aust. J. Earth Sci. 44 (5), 689-700.

Nait-Chabane, A., Zerr, B., 2014. Unsupervised Knowledge Discovery of Seabed Types using Competitive Neural Network: Application to Sidescan Sonar Images. In: OCEANS - Saint John's (CA), 2014 MTS/IEEE, pp. 1-5.

Nait-Chabane, A., Zerr, B., Chenadec, G.L., 2013. Sidescan sonar imagery segmentation with a combination of texture and spectral analysis. In: 2013 MTS/IEEE OCEANS Bergen, pp. 1-6, http://dx.doi.org/10.1109/OCEANS-Bergen.2013.6608096.

Sammon, J.W., 1969. A nonlinear mapping for data structure analysis. IEEE Trans. Comput. 18 (5), 401-409.

Stewart, W., Jiang, M., Marra, M., 1994. A neural network approach to classification of sidescan sonar imagery from a midocean ridge area. IEEE J. Ocean. Eng. 19 (2), 214-224.

Uriarte, E.A., Martin, F.D., 2005. Topology preservation in SOM. Int. J. Math. Comput. Sci. 1 (1), 19-22.

Vesanto, J., Alhoniemi, E., 2000. Clustering of the self-organizing map. IEEE Trans. Neural Netw. 11 (3), 586-600.

Vink, F.H., Hollett, R., Zerr, B., 2000. Towards bottom classfication with AUVs: Segmentation of sidescan sonar imagery using an unsupervised artificial neural network. In: Bovio, T.R., E., S.H., (Eds.), Autonomous underwater Vehicle and Ocean Modelling Networks: COATS 2000 Conference Proceeedings CP-46, pp. 209219.

Wynn, R.B., Huvenne, V.A., Bas, T.P.L., Murton, B.J., Connelly, D.P., Bett, B.J., Ruhl, H.A., Morris, K.J., Peakall, J., Parsons, D.R., Sumner, E.J., Darby, S.E., Dorrell, R.M., Hunt, J.E., 2014. Autonomous Underwater Vehicles (AUVs): their past, present and future contributions to the advancement of marine geoscience. Mar. Geol. 352 (0), 451-468.

Zadeh, L.A., 1965. Fuzzy sets, Information and Control, 8, 3, pp. 338-353.

Zahn, C., 2007. Graph-theoretical methods for detecting and describing gestalt clusters. IEEE Trans. Comput. 1 (100), 68-86. 